\title{
Artigos Retratados Sobre COVID-19: Uma Análise Sobre Sua Rede de Citações
}

\author{
Hugo Duca ${ }^{1}$, Ingrid Pacheco ${ }^{1}$, Giseli Rabello Lopes ${ }^{1}$, Maria Luiza M. Campos ${ }^{1}$, Jonice Oliveira ${ }^{1}$ \\ ${ }^{1}$ Programa de Pós-Graduação em Informática - Instituto de Computação \\ Universidade Federal do Rio de Janeiro (UFRJ) \\ Rio de Janeiro - RJ - Brasil \\ \{hugo.duca,mluiza\}@ppgi.ufrj.br, \{ingridpacheco,giseli,jonice\}@dcc.ufrj.br
}

\begin{abstract}
To help fight the COVID-19 pandemic, some researches were accelerated, resulting in many articles being hastily published, some of which were retracted. In the present work, social network analysis techniques and centrality and betweenness metrics are used to assess the influence of retracted articles on those that referred to them. As a result, it was found that the most referred retracted work had 2,745 citations, 648 articles refer to more than one retracted article and 7 retracted works refer to others in the same situation. In addition, it was found that $82.11 \%$ of the references were made after the retraction and that the most used words in the titles were associated with possible treatments for the disease.
\end{abstract}

Resumo. Para auxiliar no enfrentamento à pandemia da COVID-19, pesquisas foram aceleradas, acarretando em muitos artigos sendo publicados apressadamente, alguns destes sofrendo retratações. Neste trabalho são utilizadas técnicas de análise de redes sociais e métricas de centralidade e intermediação para avaliar a influência dos trabalhos retratados sobre os que os referenciam. Como resultado foi verificado que o trabalho retratado mais referenciado teve 2.745 citações, 648 artigos fazem referência a mais de um retratado e 7 retratados referenciam outros na mesma situação. Além disso, foi constatado que $82,11 \%$ das referências foram feitas após a retratação e que as palavras mais usadas nos títulos eram associadas aos possíveis tratamentos da doença.

\section{Introdução}

No início de 2020, a Organização Mundial da Saúde (OMS) declarou a pandemia do Corona Virus Disease 2019 (COVID-19), uma doença relativamente nova. Embora o SARS$\mathrm{CoV}$ já fosse conhecido, o SARS-CoV2 mostrou-se muito mais contagioso, e a doença mais agressiva com sintomas e características epidemiológicas ainda parcialmente desconhecidas. Assim, com rápida disseminação da doença, as pesquisas foram aceleradas e muitos de seus resultados rapidamente divulgados, na tentativa de auxiliar no enfrentamento da crise [Soltani e Patini 2020]. Ao mesmo tempo, considerando a velocidade com que as informações são divulgadas atualmente, nem sempre com sua veracidade ou fontes verificadas, o efeito de resultados imprecisos ou mal interpretados pode ser crítico.

A corrida para publicação de artigos sobre a COVID-19 trouxe preocupações acerca da qualidade metodológica e a validade científica dos mesmos. Em junho de 2020, em uma pesquisa no Retraction Database ${ }^{1}$ usando as palavras-chave:

\footnotetext{
${ }^{1}$ http://retractiondatabase.org/RetractionSearch.aspx
} 
“COVID-19”, “coronavirus disease 2019”, “coronavirus 2019”, "SARS-COV-2” e “2019nCov", foi possível encontrar 26 artigos que haviam sido removidos ou retratados [Soltani e Patini 2020].

A retratação de artigos pode ser definida como um mecanismo para corrigir a literatura e alertar os leitores sobre artigos que contenham conteúdos ou dados errôneos e que suas descobertas ou conclusões podem ser falhas. O seu principal propósito é garantir a integridade e corrigir a literatura, e não de punir os autores. [Browman et al. 2019].

Dessa forma, uma retratação indica que o trabalho em questão não deveria ter sido publicado, que suas conclusões e dados não devem ser usados para apoio a pesquisas futuras. Alguns dos motivos para retratação de um artigo são: plágio, pesquisa não confiável, dados publicados anteriormente e pesquisa antiética. Quando ocorre uma retratação, significa que ocorreu algum problema tão significativo com a pesquisa que suas descobertas podem ser invalidadas [Sheth e Thaker 2014].

Quando usada corretamente, uma citação serve como uma ferramenta valiosa para apoiar uma afirmação, método ou hipótese. Por outro lado, um trabalho que foi oficialmente marcado como retratado pelo editor foi, em essência, riscado na íntegra do registro acadêmico [Silva e Bornemann-Cimenti 2017].

Entretanto, muitos são os casos em que os artigos, mesmo após sofrerem retratação, continuam sendo usados para apoiar novas descobertas científicas, o que pode colocar em risco o processo científico. No site retractionwatch. com, pode-se observar exemplos de artigos que possuem o número de citações ainda maior após a retratação do mesmo, demonstrando a gravidade do problema mencionado.

Neste contexto, visando analisar retratações e citações na temática da COVID19, são formuladas as seguintes perguntas de pesquisa a serem respondidas pelo presente artigo:

- P1 Quais são os artigos retratados mais referenciados?

- P2 Quais os principais temas e similaridades entre os artigos retratados?

- P3 As citações ocorreram antes ou após o aviso de retratação?

- P4 Existe uma cadeia de artigos retratados (que referenciam outros na mesma condição)?

- P5 Quais são os artigos que mais referenciam artigos retratados (possivelmente diminuindo significativamente a sua qualidade)?

Visando responder as questões de pesquisa elencadas, e considerando a falta de um conjunto de dados único com as informações necessárias, foi construído, através de um script em Python, um conjunto de dados que continha 5884 trabalhos que referenciavam 73 artigos retratados na temática de COVID-19, dados suficientes para as análises propostas.

Posteriormente, foi construída uma rede com base no conjunto encontrado, e empregadas diferentes técnicas de Análise de Redes Sociais (ARS) [Wasserman e Faust 1994]. Assim, foi possível encontrar os 5 artigos retratados mais referenciados, majoritariamente após o aviso de retratação, dentre os quais um deles é citado por 2745 artigos e outro ainda referencia 2 trabalhos na mesma situação, demonstrando a existência de uma cadeia de referências de retratados. 
Ademais, foi possível descobrir que uma grande porção destes mencionava remédios que eram citados como possíveis tratamentos para a COVID-19 no início da pandemia. A partir destes resultados ficou perceptível o quão recorrente são as citações a artigos retratados, e o quanto isto pode impactar na confiabilidade das pesquisas.

O restante deste artigo está organizado da seguinte forma. Na seção 2, são discutidos os trabalhos relacionados a artigos, citações e retratações na temática da COVID-19. Na seção 3, são discutidas métricas utilizadas na análise de redes sociais. Na seção 4, são apresentadas as informações sobre a criação do conjunto de dados. Na seção 5, são apresentadas as análises realizadas e os resultados obtidos. Por fim, na seção 6, são apresentadas as conclusões e trabalhos futuros.

\section{Trabalhos Relacionados}

Nesta seção, são discutidos alguns trabalhos relacionados especificamente a artigos, retratações e citações na temática da COVID-19/SARS-CoV-2.

\subsection{Publicações e Citações}

Diferentes estudos enfatizam o crescimento das publicações sobre COVID-19 ao longo da pandemia. No final de 2020 haviam estimativas acerca da publicação de mais de 100.000 artigos e pré-prints sobre o assunto, e da possibilidade de este número ultrapassar os 200.000 em dezembro do mesmo ano [Else 2020]. Além disso, dentre as análises apresentadas estão os principais tópicos abordados (modelagem epidemiológica, controle de propagação, saúde pública, diagnósticos e testes, saúde mental e mortalidade em hospitais) e os países dos autores centrais (China e Estados Unidos) [Boschieroa et al. 2021].

Em outro estudo, foram utilizados dados coletados da PubMed ${ }^{2}$, entre março e abril de 2020, sendo realizada uma classificação de acordo com o nível de evidência para uma avaliação quantitativa de qualidade metodológica e uma análise narrativa dos pontos fortes e fracos das publicações da COVID-19 [Zdravkovic et al. 2020]. Este mostrou evidências de que a qualidade das publicações sobre o tema nos três periódicos da área médica mais bem classificados (The New England Journal of Medicine (NEJM), The Journal of the American Medical Association (JAMA), e The Lancet) está abaixo da média de qualidade desses periódicos. Os autores relataram ainda que o número de publicações sobre COVID-19 é quase igual ao número de publicações em todos os outros tópicos, destacando a importância de fomentar um debate sobre o valor científico, ética e sobrecarga de informações nas pesquisa sobre a temática.

Apesar de a publicação durante a pandemia parecer repleta de riscos, é tentadora pelo imenso interesse do público e, em comparação a outros tópicos, os artigos sobre COVID-19 demostraram gerar mais citações (mediana 45 vs 2 citações) [Cortegiani et al. 2021]. Um Estudo que usou como fonte o site Retraction Watch ${ }^{3}$ relatou que houve um aumento na velocidade das revisões em periódicos da área médica e um alarmante crescimento nas retrações de artigos relacionados à COVID-19 (15 pré-prints e 24 artigos de periódicos retirados ou retratados até dezembro de 2020)[Else 2020].

\footnotetext{
${ }^{2}$ https://pubmed.ncbi.nlm.nih.gov/

${ }^{3}$ https://retractionwatch.com/
} 


\subsection{Retratações e Citações}

Estudos recentes, feitos em Novembro de 2020, apresentam análises preliminares sobre retratações de artigos sobre COVID-19, usando as bases de dados Retraction Watch e PubMed. De acordo com eles, naquela época já existiam 39 casos de artigos retratados, encontrados tanto em periódicos bem estabelecidos quanto naqueles com fator de impacto mais baixo, tendo seus autores um $h$-index moderadamente alto [Cortegiani et al. 2021].

Dentre os principais motivos de retratações estão desde duplicatas e plágio até questões metodológicas e de má interpretação de dados [Boschieroa et al. 2021]. Duplicação, questões éticas e plágio são mais frequentes em periódicos com baixo indicador de SJR (SCImago Journal Rank), enquanto periódicos com alto indicador de SJR tem, em sua maioria, questões metodológicas como motivo de retratação.

De acordo com os levantamentos realizados, pode-se observar que grande parte dos trabalhos relacionados concentra-se em caracterizar publicações e retratações sobre o COVID-19. Em uma investigação dos 200 artigos acadêmicos mais recentes publicados em 2020, mais da metade deles, incluindo os publicados em periódicos conceituados, usaram os artigos retratados para apoiar suas descobertas científicas e não notificaram as retratações [Piller 2021]. Além disso, não foram encontrados estudos mais aprofundados em relação as redes de citações destes artigos retratados. Dessa forma, o presente trabalho visa realizar este estudo através do uso de técnicas de análises de redes sociais.

\section{Análise de Redes Sociais}

Uma rede social é definida por um conjunto finito de atores e as relações definidas sobre eles [Wasserman e Faust 1994]. A presença de informações relacionais é um aspecto crítico e definidor de uma rede social. $\mathrm{Na}$ análise de redes sociais, uma rede representa um grupo de atores (nós) que se relacionam. Essas relações, links ou vínculos (arestas) se caracterizam por fluxos de informação. Desta maneira, fluxos, nós e vínculos constituem os elementos básicos de uma rede [Ribeiro e Bastos 2011].

Dentre as métricas para análise de redes sociais estão incluídas as de centralidade. O papel da centralidade dentro de uma rede é parte do que geralmente tentamos entender durante a análise. Existem diversas maneiras de se medir a centralidade, sendo que cada uma delas serve para auxiliar em um tipo de entendimento específico [Cherven 2015]. A seguir será feita uma breve introdução sobre estas métricas, em especial, as que serão empregadas para as análises apresentadas na seção 5.

- Centralidade local: é calculada como o número total de links diretos com os demais nós da rede, consequentemente, um valor elevado de centralidade representa uma posição mais centralizada do nó. Esses nós podem ajudar a facilitar o fluxo de informações de um grupo para o outro dentro de um contexto organizacional [Hatala 2006].

- Centralidade de intermediação (Betweeneess centrality): é calculada como o número de menores caminhos entre quaisquer dois nós da rede que passam pelo determinado nó. Nós com alto valor de centralidade de intermediação podem oferecer o caminho mais direto a clusters desconectados, sendo denominados como pontes. No entanto, ser uma ponte não é um pré-requisito para ter um alto valor de de centralidade de intermediação, mas é comum que esses nós sejam classificados como criticamente importantes usando essa medida [Cherven 2015]. 
Além disso, com o propósito de prover uma melhor visualização para grafos, comumente utilizados na representação de redes sociais, alguns algoritmos foram desenvolvidos. Neste artigo foi-se optado usar o algoritmo de distribuição Fruchterman Reingold, que faz um bom trabalho em distribuir os vértices de maneira uniforme, tornando os comprimentos das bordas homogêneos e refletindo a simetria [Fruchterman e Reingold 1991].

\section{Construção do Conjunto de Dados}

Para realizar as análises propostas algumas etapas foram necessárias, incluindo a construção de um conjunto de dados que tivesse todas as informações necessárias. Os dados foram coletados de três fontes distintas: Retraction Watch Database ${ }^{3}$, para obter a listagem dos artigos retratados, Semantic Scholar ${ }^{4}$, para obter as citações e informações deles, e a DOI API ${ }^{5}$, para obter a data de publicação dos mesmos. A segunda fonte mencionada ainda foi utilizada para verificar se o trabalho que fez referência ao retratado foi fortemente influenciado pelo mesmo, dependendo do número de vezes que a referência aparece, assim como a sua localização e contexto.

Portanto, foi utilizado o Retraction Watch Database filtrando o campo de título por: "COVID-19” or "coronavirus disease 2019" or "coronavirus 2019” or "SARSCOV-2" or "2019-nCov". Através desta consulta, realizada em 19 de março de 2021, foram obtidos 92 artigos. Destes, 9 não tinham DOI disponível, restando 83 resultados, dos quais 6 estavam aparecendo repetidamente na listagem, portanto, resultando em 77 artigos retratados para análise.

Em seguida, a Semantic Scholar API $^{6}$ foi utilizada para obter mais informações sobre cada artigo retratado e os trabalhos que os citavam. Foi verificado que 4 dos artigos retratados não estavam disponíveis na API, restando 73 artigos nessa situação. Por fim, o script, através de requisições para a DOI API, obteve a data de publicação dos artigos, um dado importante para a análise e que não estava presente na requisições iniciais.

O conjunto de dados foi criado em 18 de abril de 2021 no formato $\operatorname{GraphML}^{7}$, contendo uma rede com 5.884 nós (artigos retratados e os que os referenciavam) e 6.623 arestas (citações). Dentre elas, as que representavam citações altamente influentes receberam peso 2 e as demais, peso 1. Além disso, sobre os nós e arestas, são armazenadas as informações apresentadas na Tabela 1.

Com o objetivo de que o código para construção do conjunto de dados, feito em linguagem Python, e o dataset gerado, possam ser aperfeiçoados e utilizados em outras pesquisas, eles foram desenvolvidos no formato Open Source, e encontram-se disponíveis no GitHub ${ }^{8}$.

\section{Análises e Resultados}

Após a geração do conjunto de dados, foi utilizada a ferramenta $G e p h i^{9}$ para realizar as análises referentes às perguntas de pesquisa previamente estabelecidas (ver seção 1).

\footnotetext{
${ }^{4}$ https://www.semanticscholar.org/

${ }^{5}$ https://www.doi.org/factsheets/DOIProxy.html\#rest-api

${ }^{6}$ https://api.semanticscholar.org/

${ }^{7}$ http://graphml.graphdrawing.org/

${ }^{8}$ https://github.com/ingridpacheco/RetractedArticlesDatasetGenerator

${ }^{9}$ https://gephi.org/
} 
Tabela 1. Informações sobre nós e arestas da rede

\begin{tabular}{|l|l|l|}
\hline Atributo & Para & Descrição \\
\hline id & nó & DOI do artigo, ou identificador único \\
\hline retractionDate & nó & Data de retratação, no formato YYYy-mm-dd \\
\hline publicationDate & nó & Data de publicação do artigo, no formato YYYY-mm-dd \\
\hline retracted & nó & Flag que identifica se um artigo é retratado \\
\hline name & nó & Título do artigo \\
\hline weight & aresta & Peso da aresta (se for artigo influente, aresta tem peso maior) \\
\hline afterRetraction & aresta & Flag para identificar que foi uma citação feita após a retratação \\
\hline influential & aresta & Flag para identificar uma citação influente \\
\hline
\end{tabular}

Através delas e com as métricas e filtragens disponíveis na ferramenta, descobertas relevantes sobre os artigos retratados puderam ser vislumbradas.

\subsection{Caracterização da Rede de Citações}

O estudo da estrutura da rede é uma etapa importante para o entendimento do tipo de análise que pode ser realizada, e neste caso, possibilita uma visão geral da problemática da citação de artigos retratados. A rede possui algumas sub-redes ego centradas (egocentered network), onde o nó central (ego) de cada sub-rede é um artigo retratado e o conjunto de nós que o cercam (alter) são artigos que fazem referência ao ego. Esse tipo de rede pode ser usada em diversas aplicações, como a análise de transmissão de doenças, estudos de suporte social e redes de discussão [Wasserman e Faust 1994].

Outro aspecto interessante é que, dos 5.884 nós da rede, 73 representam artigos que sofreram retratação e os outros 5.811 nós restantes são os artigos que os citam. Em relação às arestas, a rede é formada por 6.623, das quais 423 têm o atributo influential igual a 1, o que significa que pelo menos $6,39 \%$ dos artigos desta rede foram fortemente influenciados pelos artigos retratados. Além disso, o número médio de citações (arestas) por artigo retratado (nó) é de 90,72.

Por fim, as características principais da rede são: grau médio de 1,126 e coeficiente de clusterização igual a 0,18 (estes valores baixos são esperados, uma vez que esta rede inclui apenas as citações a artigos retratados). Os números de componentes fracamente e fortemente conectados são, respectivamente, 39 e 5.884. Na Figura 1 pode-se ver uma representação do grafo da rede completa usando o algoritmo de visualização Fruchterman-Reingold. Cada nó da rede representa um artigo, estando os retratados em vermelho e o tamanho de cada nó sendo diretamente proporcional ao seu grau (centralidade local). Por fim, as arestas representam as referências aos artigos retratados, estando em verde as arestas que ligam os artigos que foram fortemente influenciados ao artigo retratado (ver seção 4).

\subsection{Artigos Retratados Mais Referenciados}

Apesar de todos os artigos retratados terem um grande impacto sobre a validade dos que os referenciam, compreender os mais citados serve para dar uma visão mais concreta e estreita sobre os principais temas que causam retratação dentro da temática do COVID-19, além de elucidar o seu impacto e a forma como a rede está disposta. 


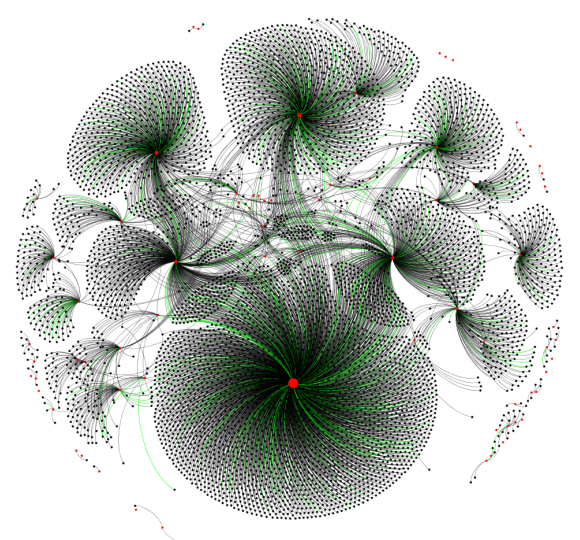

Figura 1. Rede de Citações de Artigos Retratados sobre COVID-19
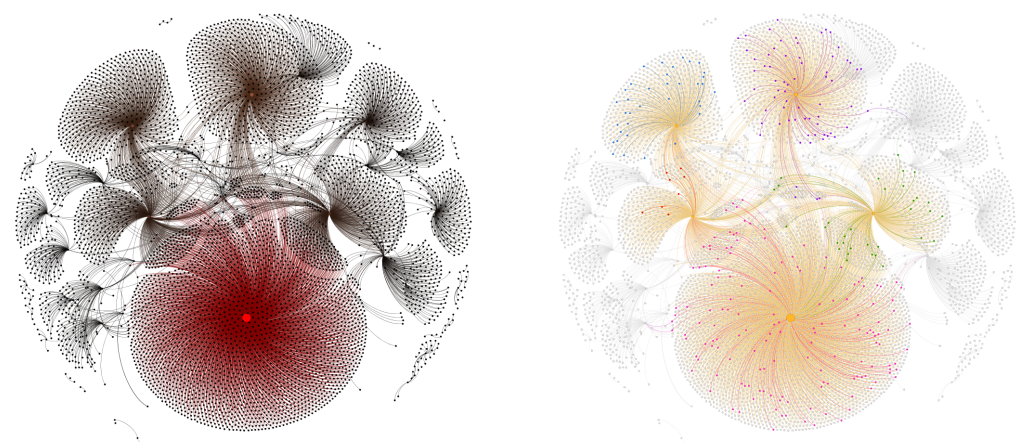

Figura 2. Rede: (a) com a métrica de centralidade; (b) de retratados com seus nós influentes

Levando em consideração este contexto, e para responder a P1 uma métrica que se mostrou interessante para esta análise foi a de centralidade local, com a finalidade de encontrar os artigos retratados que fossem mais referenciados. Ao aplicar tal métrica sobre a rede social, o grafo da Figura 2 (a) foi gerado. O nó em destaque, na cor vermelha, representa o artigo com maior valor de centralidade local, correspondente ao artigo retratado mais citado (referenciado por 2.745 artigos), de título Hydroxychloroquine and azithromycin as a treatment of COVID-19: results of an open-label non-randomized clinical trial e DOI 10.1016/j.ijantimicag.2020.105949. Levando em consideração o fato de que o segundo artigo retratado com maior centralidade é citado por 799 artigos, esse valor passa a ser ainda mais significativo, expressando o quanto o artigo mais referenciado é influente dentro da rede.

Estendendo as análises, os 5 artigos retratados mais referenciados foram filtrados, ainda utilizando a métrica de centralidade local, a fim de tentar encontrar similaridades entre eles. A Figura 2 (b) representa os 5 principais artigos retratados na cor amarela e suas citações influentes (que possuem o atributo influential na aresta de ligação igual a 1) em diferentes cores, dependendo do artigo que referenciam.

A Tabela 2 apresenta informações sobre os top 5 artigos retratados mais citados. 

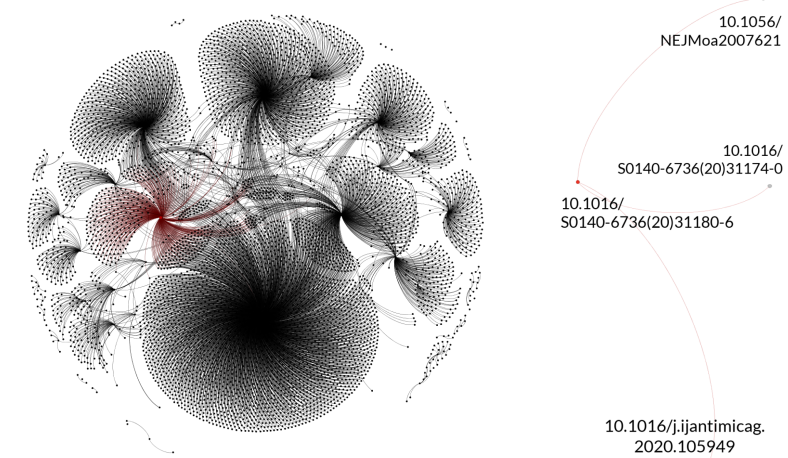

Figura 3. Rede: (a) destacando métrica de intermediação; (b) ego centrada no nó com maior valor de intermediação

Salienta-se que a retratação do primeiro artigo ocorreu por problemas em relação aos seus resultados, o que possivelmente prejudicou os 150 artigos que foram fortemente influenciados por ele, consequentemente, tendo suas validações questionadas. O segundo foi publicado no National Science Review e teve como principal motivo de retratação erro no texto, o que também pode acabar afetando a qualidade dos artigos que o referenciam. O terceiro foi publicado no The New England journal of medicine e os motivos de sua retratação se expandem, não só tendo incertezas nos resultados como também nos dados utilizados, mostrando os problemas que referenciá-lo pode causar. O quarto, publicado no Engineering, não teve o seu motivo de retratação muito claro, apenas tendo a indicação de que o artigo foi retirado, sem maiores informações sobre o que o levou a essa situação. Por fim, o quinto artigo, publicado no The Lancet, é citado por outro retratado, 10.1016/S0140-6736(20)31174-0, e ainda é um dos 7 retratados que cita outros na mesma condição. Inclusive, dentre eles, este é o único que cita mais de um retratado, neste caso, sendo os artigos 10.1016/j.ijantimicag.2020.105949 e 10.1056/NEJMoa2007621. Devido a este fato, o artigo cria uma ponte entre dois trabalhos retratados muito relevantes na rede, e fica com o maior valor de intermediação (711.833), como visto na Figura 3 (a). Ademais, este artigo e suas principais conexões são destacados na Figura 3 (b).

Passando para a análise da rede como um todo, um dos primeiros pontos que podese notar é um tema em comum que aparece nos títulos de $75 \%$ deles: tratamento para a COVID-19. A fim de responder a P2, análises sobre a incidência de termos nos títulos dos artigos, usando a ferramenta WordArt.com ${ }^{10}$, foram feitas considerando: (a) todos os artigos da rede e (b) apenas do subgrupo dos 73 artigos retratados. Foram executadas as seguintes etapas de pré-processamento: remoção de stopwords, remoção de números e stemming [Baeza-Yates e Ribeiro-Neto 2013]. Além destas, também foram removidos os termos utilizados para a coleta dos dados no Retraction Watch Database (ver seção 4). Os resultados dessas análises estão sumarizados na Tabela 3. Dentre as palavras-chave mais frequentes nos dois grupos, pode-se evidenciar Hydroxychloroquine, Treatment e Drug. Estes termos se destacam devido ao contexto atual da pandemia da COVID-19, momento em que são divulgados estudos relacionados ao tratamento da mesma.

Além disso, outra análise sobre a rede de citações de artigos retratados foi condu-

\footnotetext{
${ }^{10}$ https://wordart.com/
} 
Tabela 2. Informações sobre citações dos top 5 artigos retratados

\begin{tabular}{|l|c|c|}
\hline Título/DOI & Citações & $\begin{array}{l}\text { Citações } \\
\text { de Influentes }\end{array}$ \\
\hline $\begin{array}{l}\text { Hydroxychloroquine and azithromycin as a } \\
\text { treatment of COVID-19: results of an open-label } \\
\text { non-randomized clinical trial } \\
\text { 10.1016/j.ijantimicag.2020.105949 }\end{array}$ & 2.745 & 150 \\
\hline $\begin{array}{l}\text { On the origin and continuing evolution } \\
\text { of SARS-CoV-2 } \\
\text { 10.1093/nsr/nwaa036 }\end{array}$ & 799 & 68 \\
\hline $\begin{array}{l}\text { Cardiovascular Disease, Drug Therapy, } \\
\text { and Mortality in Covid-19 } \\
\text { 10.1056/NEJMoa2007621 }\end{array}$ & 627 & 36 \\
\hline $\begin{array}{l}\text { Experimental Treatment with Favipiravir } \\
\text { for COVID-19: An Open-Label Control Study } \\
\text { 10.1016/j.eng.2020.03.007 }\end{array}$ & 508 & 33 \\
\hline $\begin{array}{l}\text { RETRACTED: Hydroxychloroquine or chloroquine with or } \\
\text { without a macrolide for treatment of COVID-19: } \\
\text { a multinational registry analysis } \\
\text { 10.1016/S0140-6736(20)31180-6 }\end{array}$ & 471 & 8 \\
\hline
\end{tabular}

zida com o intuito de considerar o aspecto temporal das citações, verificando a quantidade de referências feitas antes e após os avisos de retratação. A Tabela 4 apresenta os quantitativos de citações antes e após retratações para os cinco artigos mais citados na rede. Já a Figura 4 ilustra a rede, destacando em amarelo as citações efetuadas (a) antes e (b) após as retratações. Como demonstrado, para os cinco artigos mais citados, o número de citações após a retratação foi expressivamente maior do que as citações anteriores ao aviso de retratação. Desta forma, 82,11\% de todas as citações da rede aconteceram após, e apenas 17,89\% ocorreram antes, respondendo assim a $\mathbf{P 3}$.
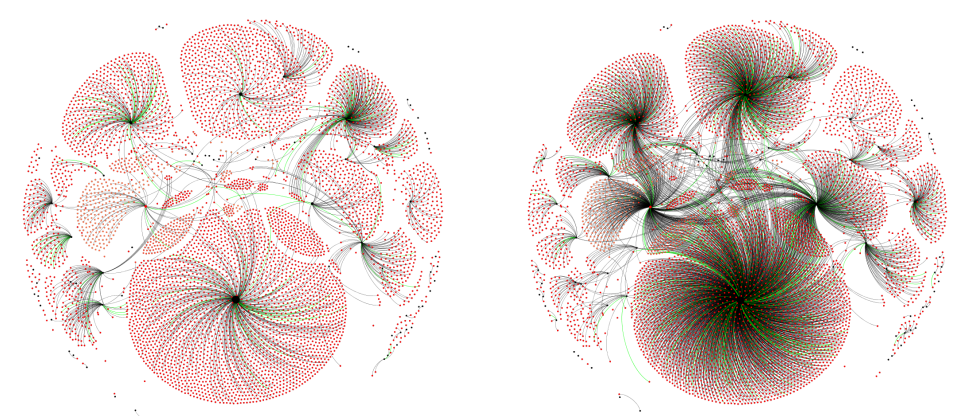

Figura 4. Rede destacando: citações feitas (a) antes e (b) após retratações; (c) artigos que mais referenciam retratados 
Tabela 3. Comparativo entre palavras-chave de todos os artigos da rede versus as palavras-chave dos artigos retratados

\begin{tabular}{|l|l|c|l|l|}
\hline \multicolumn{2}{|c|}{ Todos os Artigos } & x & \multicolumn{2}{c|}{ Artigos Retratados } \\
\hline Termo & Peso & & Termo & Peso \\
\hline Patient & 893 & $\mathbf{1}^{\circ}$ & Patient & 20 \\
\hline Review & 717 & $\mathbf{2}^{\circ}$ & Pandemic & 9 \\
\hline Pandemic & 663 & $\mathbf{3}^{\circ}$ & Infect & 8 \\
\hline Treatment & 629 & $\mathbf{4}^{\circ}$ & Analysis & 7 \\
\hline Hydroxychloroquine & 601 & $\mathbf{5}^{\circ}$ & Clinic & 6 \\
\hline Infect & 576 & $\mathbf{6}^{\circ}$ & Effect & 6 \\
\hline Clinic & 524 & $\mathbf{7}^{\circ}$ & Treatment & 6 \\
\hline Drug & 438 & $\mathbf{8}^{\circ}$ & Hospital & 5 \\
\hline Analysis & 416 & $\mathbf{9}^{\circ}$ & Hydroxychloroquine & 5 \\
\hline Study & 380 & $\mathbf{1 0}^{\circ}$ & Mortality & 5 \\
\hline
\end{tabular}

Tabela 4. Citações antes e após retratação para os top 5 artigos retratados

\begin{tabular}{|l|c|c|c|}
\hline Título & Data Retratação & Antes & Após \\
\hline $\begin{array}{l}\text { Hydroxychloroquine and azithromycin as a } \\
\text { treatment of COVID-19: results of an open-label } \\
\text { non-randomized clinical trial }\end{array}$ & $11 / 04 / 2020$ & 260 & 2485 \\
\hline $\begin{array}{l}\text { On the origin and continuing evolution } \\
\text { of SARS-CoV-2 }\end{array}$ & $19 / 06 / 2020$ & 49 & 750 \\
\hline $\begin{array}{l}\text { Cardiovascular Disease, Drug Therapy, } \\
\text { and Mortality in Covid-19 }\end{array}$ & $04 / 06 / 2020$ & 117 & 510 \\
\hline $\begin{array}{l}\text { Experimental Treatment with Favipiravir } \\
\text { for COVID-19: An Open-Label Control Study }\end{array}$ & $01 / 04 / 2020$ & 30 & 478 \\
\hline $\begin{array}{l}\text { RETRACTED: Hydroxychloroquine or chloroquine } \\
\text { with or without a macrolide for treatment of COVID-19: } \\
\text { a multinational registry analysis }\end{array}$ & $04 / 06 / 2020$ & 36 & 435 \\
\hline
\end{tabular}

\subsection{Artigos Que Mais Referenciam Retratados}

Em resposta a $\mathbf{P 4}$, há 7 retratados que referenciam artigos na mesma situação. Além disso, foi feita uma análise para encontrar quais eram os artigos que mais citavam retratados. Ao analisar a rede procurando por estes nós, filtrando os que referenciavam mais de um retratado, foi possível encontrar 648 artigos (já considerando o quinto artigo retratado mais citado mencionado na seção anterior), desta maneira respondendo a P5. Porém, os 3 nós que mais tiveram conexões, destacados em vermelho, mostrados na Figura 4 (c), possuem um diferencial em relação aos outros devido à quantidade de retratados que eles citam. Enquanto o quarto lugar referencia apenas 5 artigos, os 3 principais, 10.1136/medethics-2020-106494, 10.1007/s11192-020-03661-9 e 10.1016/j.pulmoe.2020.10.011, referenciam uma quantidade significativa de artigos retratados, respectivamente, 19,17 e 16.

O grande número chama a atenção para o tema em comum que eles tratam, pois todos abordam o assunto de artigos retratados sobre COVID-19 como uma forma de crítica 
para a velocidade que as publicações são realizadas, ao invés de priorizar a qualidade e a busca por informações corretas, portanto, fazendo as referências propositalmente para trazer exemplos que elucidem o problema.

Dentre eles, entretanto, vale mencionar que o artigo de DOI 10.1007/s11192-02003661-9, que possui o título de Retracted COVID-19 articles: a side-effect of the hot race to publication [Soltani e Patini 2020], é o único que cita todos os 5 retratados mencionados antes, evidenciando o quão relevante ele é para o tema e para a rede social utilizada.

\section{Conclusões}

Com o avanço das descobertas científicas, o compartilhamento de todos os resultados encontrados no menor tempo possível é essencial. Entretanto, essa pressa por publicações pode implicar em diversos problemas com relação a qualidade do artigo, o levando a retratação. Portanto, mecanismos que analisem os artigos retratados ou auxiliem na hora de referencia-los se mostram muito importantes, principalmente diante do conhecimento adquirido por este trabalho.

Mediante o exposto, é possível afirmar que através da ARS foi possível descobrir os artigos retratados mais relevantes no contexto da COVID-19. Usando as métricas de centralidade este trabalho mensurou a influência dos trabalhos retratados em artigos válidos ou não. Ademais, foi possível identificar pesquisas que sofreram retratações e faziam referência a artigos na mesma situação. Por fim, também foi verificado que nesta rede a maior parte das citações foram feitas após o aviso de retratação.

Considerando o fato, esse trabalho demonstra a importância de uma maior discussão sobre o tema que hoje não é tão difundido como deveria. Além disso, foi detectado que as palavras mais utilizadas nos títulos destes trabalhos referiam-se a possíveis tratamentos e medicamentos relacionados a doença, deixando em aberto a possibilidade de que essas publicações influenciem de alguma forma a disseminação das notícias falsas.

No entanto, durante o desenvolvimento deste trabalho alguns desafios foram encontrados. O principal foi a falta de um conjunto de dados único com informações sobre as retratações e citações ou referências feitas por esses artigos. Para mitigar este, foi necessário o desenvolvimento de um script em Python que, coletando dados de três bases distintas, criasse a rede de citações de artigos retratados a ser utilizada nas análises.

Como trabalhos futuros, pretende-se ampliar essas análises em uma rede completa sobre a COVID-19, também com as conexões entre artigos não retratados, diferentemente da usada nesse trabalho (ego-centered), que foi gerada a partir dos retratados. Desta forma, seria possível verificar mais amplamente o quão influentes realmente são os artigos retratados. Além disso, pretendemos realizar a automatização de parte das análises afim de evitar possível subjetividade nos resultados. Finalmente, uma outra abordagem interessante seria o cruzamento da rede de retratações com uma rede de fake news associadas à pandemia da COVID-19, a fim de encontrar possíveis conexões entre as notícias falsas e as publicações retratadas (como provável fontes delas).

\section{Referências}

Baeza-Yates, R. and Ribeiro-Neto, B. (2013). Recuperação de Informação: Conceitos e Tecnologia das Máquinas de Busca. 2nd edition. Bookman. 
Boschieroa, M. N., Carvalho, T. A., and de Lima Marson, F. A. (2021). Retraction in the era of covid-19 and its influence on evidence-based medicine: is science in jeopardy? Pulmonology Journal, 27:97-106.

Browman, H., Alexander, J., Fennell, C., Hodgkinson, M., and Tierney, H. (2019). Guidelines for retracting articles. Technical report, Committee on Publication Ethics.

Cherven, K. (2015). Mastering Gephi network visualization : produce advanced network graphs in Gephi and gain valuable insights into your network datasets. Community experience distilled. Packt Publishing.

Cortegiani, A., Catalisano, G., Ippolito, M., Giarratano, A., Absalom, A. R., and Einav, S. (2021). Retracted papers on sars-cov-2 and covid-19. British journal of anaesthesia, 126:e155-e156.

Else, H. (2020). How a torrent of covid science changed research publishing - in seven charts. Nature, 588:553.

Fruchterman, T. M. J. and Reingold, E. M. (1991). Graph drawing by force-directed placement. Software: Practice and Experience, 21(11):1129-1164.

Hatala, J.-P. (2006). Social network analysis in human resource development: A new methodology. Human Resource Development Review, 5(1):45-71.

Piller, C. (2021). Disgraced covid-19 studies are still routinely cited. Science, 371:331332.

Ribeiro, E. M. B. d. A. and Bastos, A. A. V. A.-1. B. (2011). Redes sociais interorganizacionais na efetivação de projetos sociais. Psicologia \& Sociedade, 23:282 - 292.

Sheth, B. P. and Thaker, V. S. (2014). Scientific retraction: a synonym for pseudoscience? Acta bioethica, 20.

Silva, J. A. T. D. and Bornemann-Cimenti, H. (2017). Why do some retracted papers continue to be cited? Scientometrics, 110:365-370.

Soltani, P. and Patini, R. (2020). Retracted covid-19 articles: a side-effect of the hot race to publication. Scientometrics, 125.

Wasserman, S. and Faust, K. (1994). Social network analysis: Methods and applications, volume 8 . Cambridge university press.

Zdravkovic, M., Berger-Estilita, J., Zdravkovic, B., and Berger, D. (2020). Scientific quality of covid-19 and sars cov-2 publications in the highest impact medical journals during the early phase of the pandemic: A case control study. PLoS One, 15. 